\title{
Análisis del tiempo de vida útil en la elaboración de mermelada de higuerón (Cucúrbita Odorífera Vell) con zanahoria (Daucus Carota)
}

\author{
Time analysis of life in the \\ development of marmalade \\ higuerón (Cucurbita Odorifera Vell) \\ with carrot (Daucus Carota)
}

\begin{abstract}
The aim of this study was to determine the lifespan in making jam by higueron and carrot mixture, motivated by the lack of a number of species of tubers and vegetables, as evidenced by the low consumption of these foods and the lack of appropriate technology for processing the same. The two raw materials are high in vitamins and minerals, higueron suitable for their characteristics and chemical composition is considered a fruitvegetable, it is possible it industrialization similar to those made with fruits, like the carrot is a food products excellent from the nutritional point of view, contributing to improve the quality of the jam. The treatments were kept under ambient conditions for 40 days when $\mathrm{pH}$ were recorded - soluble solids ( ${ }^{\circ}$ Brix) Heartburn - Microbiological and organoleptic analysis in order to identify the best experimental treatment tests. According to the results obtained and microbiological analysis establishes a lifetime about 6 months.
\end{abstract}

Key words: Processing vegetables, manufacture of vegetables, higueron, carrot, nutrients, marmalade.

\section{INTRODUCCIÓN}

Las propiedades beneficiosas de las frutas y vegetales sobre la salud se asocian con los metabolitos secundarios llamados fitonutrientes, los cuales pueden ser agrupados como fenoles, terpenos y derivados azufrados [1]. Su uso es variado entre los cuales consta uso digestivo, calórico y antiparasitario [2].

El higuerón aporta carbohidratos, vitaminas ( $A, B 2$; a-tocopherol, $C$ y E), aminoácidos, flavonoides y minerales $(\mathrm{K}+, \mathrm{Ca} 2+, \mathrm{P})$, además tiene un bajo contenido energético (17 Kcal por 100 g de pulpa) [3]. Las zanahorias, oriundas del Oriente (Afganistán) y Asia Central [4] son apreciadas por su valor nutricional a través de los carotinoides como un aporte de provitamina A. [5]

La zanahoria es una raíz importante para la industria de conserva, y se destinan cantidades considerables a enlatar
Fernando Álvarez Calvache (1) Edwin Santamaría D. (2) Edwin Santamaría F. (2) Estefanía Lara A. (1)

(1) Facultad de Ciencias e Ingeniería en Alimentos, Universidad Técnica de Ambato, Ambato, Ecuador (2) Facultad de Ciencias Administrativas, Universidad Técnica de Ambato, Ambato, Ecuador

Dirigir la correspondencia a: Profesor

Edwin Santamaría Freire Máster en Estadística, Docente Investigador, Universidad Técnica de Ambato, Ecuador. Río Cutuchi y Av. Los Chasquis, Cód: 180208 , 593998011233 e-mail: edwinjsantamaria@uta.edu.ec

Este trabajo fue recibido el 5 de Abril de 2016 y aceptado para ser publicado el 2 de Julio de 2016.

y congelar. Las variedades de zanahorias son más de cinco categorías [6], con un alto contenido de agua y bajo aporte energético destacándose el aporte de carbohidratos, niacina, potasio y sodio. [7]

La explotación de los frutos nativos es reducida en Ecuador, limitando así su importancia económica [8]. Tradicionalmente, la mayor parte de las frutas que llega a los mercados y ferias locales proviene de pequeños huertos de baja productividad, carentes en general de técnicas que posibiliten un mejor rendimiento. Sin embargo, a nivel mundial existen referentes de cosecha en América, Europa y Asia [9].

Actualmente, se busca cambiar esta realidad y generar líneas de industrialización de diversas hortalizas, frutas y verduras como lo estipula el objetivo del país: "generar un cambio en la matriz productiva". Hay existencia de producción de hortalizas y se requiere generar valor agregado, es por ello 
que se establece como pregunta de investigación: ¿El tiempo de vida útil de la mermelada del higuerón (cucúrbita odorífera Vell) con zanahoria amarilla (daucus carota) será lo suficientemente largo para su conservación?

El objetivo de la investigación fue desarrollar un nuevo producto nutricional con sabor apetecible analizando su tiempo de vida útil en la elaboración de mermelada mediante una mezcla de higuerón (cucúrbita odorífera vell) con zanahoria (daucus carota), brindando un producto novedoso y que ayude en la salud debido a las propiedades que posee tal hortaliza.

Se genera la hipótesis: El tiempo de vida útil de la mermelada del higuerón con zanahoria amarilla como proceso de conservación es superior al tiempo establecido en la Norma INEN 419 [10].

\section{MATERIAL Y MÉTODOS}

Se utiliza los principios de investigación metodológicos del positivismo y neopositivismo, que contemplan el desarrollo de estándares de diseño estrictos antes de iniciar la investigación y estudiar propiedades y fenómenos cuantitativos y sus relaciones, [11] así como revisar la teoría existente, estudiar la asociación o relación entre variables cuantificadas [12].

La investigación se realizó en dos etapas: cuantitativa, a través del análisis de resultados de pruebas físico químicas y biológicas; $y$, cualitativa por estudio sensorial para verificar el estudio de vida útil del producto a través de la obtención de datos a partir de varios análisis, [13] determinando de esta manera el mejor tratamiento que se pueda utilizar dentro del diseño experimental. Para establecer la relación entre los factores de estudio dentro del diseño experimental [14], se estableció la relación de pulpa de higuerón - azúcar y cantidad de zanahoria, para ello se aplicó diseño de dos factores y tres niveles, es decir $3^{n}$ por lo que tendrá 9 tratamientos, los mismos que al trabajar con una réplica da un total de 18 tratamientos. Aplicando el siguiente modelo matemático:

$$
Y_{i j k}=\mu+A_{i}+B_{j}+(A B)_{i j}+R_{k}+\varepsilon_{i j}
$$

Para la evaluación sensorial se escogerán catadores semientrenados en forma aleatoria, aplicando un diseño de bloques incompletos [15], bajo el siguiente modelo matemático:

$$
Y_{i j}=\mu+T_{i}+B_{j}+\varepsilon_{i j}
$$

En el análisis sensorial, se utilizó cataciones donde se aplicó diseño experimental de bloques incompletos, asignando 3 tratamientos a cada catador, existiendo 15 catadores semientrenados.

Las respuestas experimentales obtenidas son:

- $\quad$ En el proceso de elaboración: La relación de cantidad de higuerón - zanahoria

- En la materia prima: análisis físico - químico, sólidos solubles ( ${ }^{\circ}$ Brix), $\mathrm{pH}$

- En la mermelada elaborada: análisis sensorial, análisis físico - químicos, $\mathrm{pH}$, sólidos solubles ( ${ }^{\circ}$ brix), acidez titulable. siguiendo la Norma 389.

- $\quad$ En el mejor tratamiento: análisis microbiológico, recuento de mohos y levaduras, vida útil, utilizando la norma INEN 386.

\section{FIGURA 1}

Diagrama de flujo de elaboración de mermelada de higuerón con zanahoria.

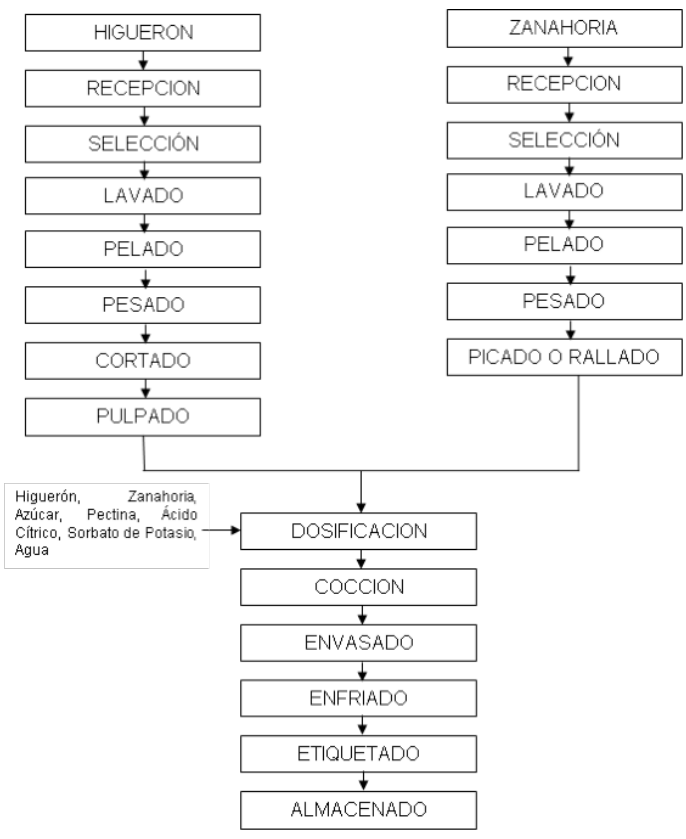


Elaboración de la mermelada

El proceso de elaboración se desarrolló considerando las normas de producción de mermelada como se presenta en la figura 1. Diagrama de flujo "Elaboración de mermelada de higuerón con zanahoria", donde se observa el ingreso de materias primas hasta el almacenamiento, para iniciar las pruebas de vita útil del producto terminado.

Se comprobó el buen estado de la fruta y se almacenó en cámara de frio a $5^{\circ} \mathrm{C}$. Tras la selección y clasificación, se eliminaron frutas podridas o deterioradas, se eliminaron manualmente las partes verdes de las zanahorias se lavaron y rallaron.

A continuación, se cortaron y pelaron los higuerones, en la última fase, se blanqueó por 10 minutos y se procedió a pulpar. Luego de poner a un lado la espuma se procedió a llenar los envases, que previamente habían sido esterilizados en agua hirviendo, se taparon a continuación para que se forme el vacío en el espacio libre.

Se prepara mermelada con la dosificación presentada en la tabla 1. Los materiales utilizados para el desarrollo de la mermelada conforme la Norma NTE INEN 2825 para las confituras, jaleas y mermeladas.

\section{RESULTADOS Y DISCUSIÓN}

Los resultados obtenidos se analizaron estadísticamente en los programas STATGRAPHICS Centurión 15.2.14; bajo el diseño experimental $3 n$ que corresponden a: cantidad (\%) de higuerón y cantidad (\%) de zanahoria como factores de estudio por medio de análisis de Varianza (Prueba de Tukey en nivel de significancia del 5\%). Las distintas determinaciones se realizaron en el Laboratorio de Biotecnología y el Laboratorio de Procesos, de la Facultad de Ciencia e Ingeniería en Alimentos, de la Universidad Técnica de Ambato (UTA).

Diámetros de las hortalizas

Análisis físico químico de la mermelada

$\mathrm{El} \mathrm{pH}$ de las mermeladas en estudio se encuentra entre valores de 3.0 a 3.4 es decir el pH de los tratamientos no se modificó drásticamente en las réplicas, y los límites establecidos por el son $(2,8-3,8),[10]$ por lo que se encuentran dentro de la norma establecida. La cual indica que: si el producto se

TABLA 1

Componentes de mermelada.

\begin{tabular}{lc}
\hline Componente & Dosificación \\
Higuerón & $27,00 \%$ \\
Zanahoria amarilla & $18,00 \%$ \\
Azúcar & $53,55 \%$ \\
Pectina & $0,40 \%$ \\
Ácido cítrico & $1,00 \%$ \\
Ácido sórbico & $0,05 \%$ \\
\hline
\end{tabular}

TABLA 2

Características de hortalizas.

\begin{tabular}{|c|c|c|c|c|}
\hline \multirow[b]{2}{*}{ Diámetro } & \multicolumn{2}{|c|}{ Higuerón } & \multicolumn{2}{|c|}{ Zanahoria } \\
\hline & R1 (cm) & $\mathrm{R} 2(\mathrm{~cm})$ & $\mathrm{R} 1(\mathrm{~cm})$ & $\mathrm{R} 2(\mathrm{~cm})$ \\
\hline Promedio & 9,07 & 8,97 & 3,63 & 3,48 \\
\hline Desviación estándar & 0,48 & 0,77 & 0,63 & 0,63 \\
\hline Peso & R1 (gr) & R2 (gr) & R1 (gr) & $\mathrm{R} 2(\mathrm{gr})$ \\
\hline Promedio & 991,48 & 943,02 & 140,61 & 143,86 \\
\hline Desviación estándar & 83,91 & 62,87 & 17,86 & 14,69 \\
\hline
\end{tabular}

TABLA 3

Análisis de varianza para $\mathrm{pH}$ de mermelada.

\begin{tabular}{llllll}
\hline Fuente & Suma de Cuadrados & Gl & Cuadrado Medio & Razón-F & Valor-P \\
EFECTOS PRINCIPALES & & & & & \\
Replicas & 0,0000888889 & 1 & 0,00008888889 & 0,02 & 0,8852 \\
RESIDUOS & 0,0320111 & 8 & 0,00400139 & & \\
TOTAL & 0,1678 & 17 & & & \\
\hline
\end{tabular}


encuentra en este rango, está protegido contra el ataque de microorganismos ya que estos no crecen en este $\mathrm{pH}$. El análisis de varianza mostró que ninguna de los réplicas tiene efecto estadísticamente significativo.

Sólidos solubles ( ${ }^{\circ}$ brix) en la mermelada

Se presentan los valores de sólidos solubles obtenidos de los diferentes tratamientos de mermelada, estos valores se encuentran en un rango de 65 a 690B para la réplica uno, mientras que para la réplica dos de 65 a $66^{\circ}$ Brix. Según norma INEN los valores de sólidos solubles para conservas vegetales debe poseer como mínimo $65^{\circ}$ Brix, por lo tanto, todos los tratamientos cumplen con lo estipulado.

Los valores de acidez de las mermeladas se encontraron en valores de 1,21 hasta 1,7 $\mathrm{g} / \mathrm{cm} 3$ para le replica uno, mientras que para la réplica dos los valores oscilan de 1,30 hasta 1,62 $\mathrm{g} / \mathrm{cm} 3$. Se determinó por refractometría en escala $(0-80)$ brix, donde se encontró valor promedio de $660 \mathrm{~B}$ y el análisis de varianza demostró que ninguno de los tratamientos tiene un efecto estadísticamente significativo.

Acidez de la mermelada

Por valoración potenciométrica con hidróxido de sodio $0,1 \mathrm{~N}$ se encontró un valor promedio de 1,44 y el análisis de varianza demostró que ninguno de los tratamientos tiene efecto estadísticamente significativo.

\section{Análisis sensorial}

Los resultados del análisis sensorial fueron medidos a través de la prueba de Tukey HSD con un nivel de significancia del $5 \%$ que mostró el tratamiento 4 con la mezcla del $30 \%$ de higuerón y $20 \%$ de zanahoria reunió las mejores características con la siguiente media en cada elemento.:

- Color. 4,883.

- Olor. 4,884

- Sabor. 5,013

- Textura. 4,252

- Aceptabilidad. 4,883

Análisis microbiológico

Se determinó un valor de (2 - 10) ufc/g en cuanto a mohos y levaduras, de esta manera se estableció un tiempo de vida útil aproximado de 6 meses.

\section{Tiempo de vida útil}

Considerando los resultados microbiológicos y siguiendo modelos propuesto para valoración sensorial por Santosa (16, 17) se desarrolla la ecuación en base a la modelización de

\section{TABLA 4}

Análisis de varianza para acidez de mermelada.

\begin{tabular}{|c|c|c|c|c|c|}
\hline Fuente & Suma de Cuadrados & $\mathrm{Gl}$ & Cuadrado Medio & Razón-F & Valor-P \\
\hline \multicolumn{6}{|l|}{ EFECTOS PRINCIPALES } \\
\hline Replicas & 0,00405 & 1 & 0,00405 & 0,28 & 0,6119 \\
\hline RESIDUOS & 0,1163 & 8 & 0,0145375 & & \\
\hline TOTAL (CORREGIDO) & 0,33045 & 17 & & & \\
\hline
\end{tabular}

\section{CALCULO DEL TIEMPO DE VIDA UTIL}

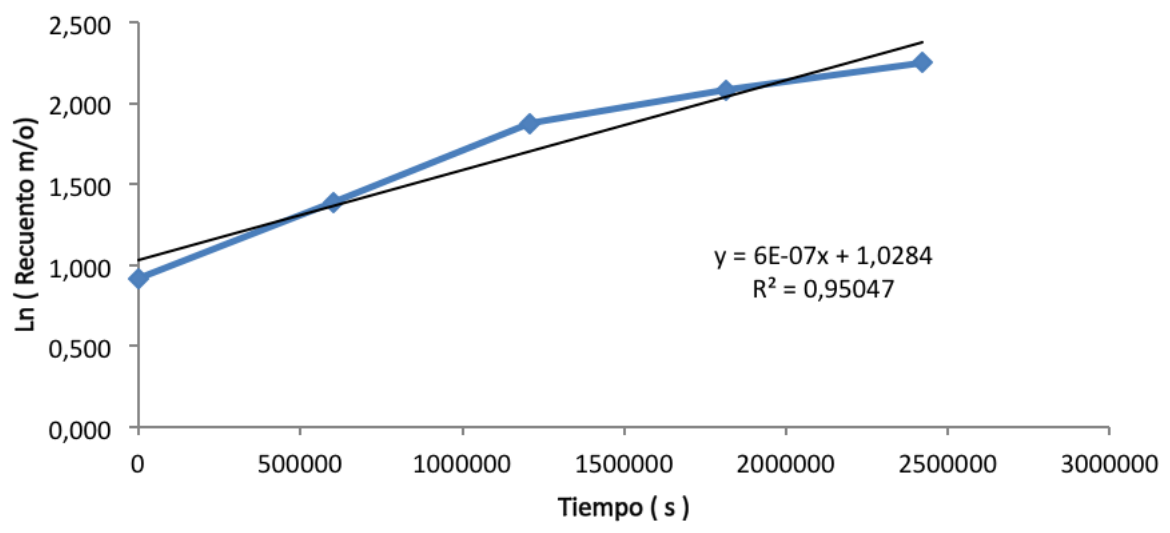


regresión lineal, de lo cual se obtiene:

$$
\operatorname{Ln}(C)=6 e^{-7} t+1,0284
$$

$$
\begin{aligned}
& R^{2}=0,9505 \\
& \operatorname{LnCo}=1,0284 \\
& K=6 e^{-7}
\end{aligned}
$$

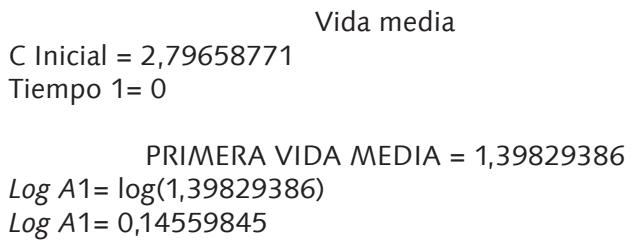

\section{TIEMPO 2}

$\operatorname{Ln}(1,39829386)=6 e^{-07 t}+1.0284$

Tiempo2 $=1155245,3$

$$
\text { SEGUNDA VIDA MEDIA }=0,69914693
$$

$\log A 2=\log (0,69914693)$

$\log A 2=-0,3579436$

\section{TIEMPO 3}

$\operatorname{Ln}(0,69914693)=6 e^{-07 t}+1.0284$

Tiempo3 $=2310490,6$

$$
\begin{gathered}
n=\frac{\log (t 3-t 2)-\log (t 2-t 1)}{\log A 1-\log A 2}+1 \\
n=\frac{\log (2310490,0-1155245,3)-\log (1155245,3-0)}{0,14559845-(-0,35789436)}+1 \\
n=1
\end{gathered}
$$

Como resultado se obtiene que $\mathrm{n}=1$ lo que quiere decir que es una ecuación de cinética de primer orden, para lo cual se procede al cálculo de vida útil.

DATOS:

$$
L n C=K t+L n C o
$$

$\operatorname{LnCo}=1,0284$

$K=6 e^{-7}$

$C=100 u f c / g$

Valores prestablecidos conociendo que las conservas comienzan a deteriorarse a partir de una carga microbiana que exceda este valor según industrias de conservas alimenticias y la Norma INEN 419 de "Conservas Vegetales - Mermelada de frutas", que establece como máximo $30 \%$ campos positivos.

$$
\begin{gathered}
\ln \frac{C}{C o}=K t \\
\ln (C)-\ln (C o)=K t \\
t=\frac{\ln (C)-\ln (C o)}{\mathrm{K}} \\
t=5,3 \text { meses }
\end{gathered}
$$

Por lo tanto, el tiempo de vida útil para la mermelada de higuerón con zanahoria va a ser alrededor de 5 a 6 meses, pese a que el producto fue elaborado con las debidas condiciones asépticas, probablemente por la utilización de materia prima poco ácida (higuerón y zanahoria). Por lo cual, se considera con Coeficiente de Determinación del 95,05\% que evidencia estadísticamente significativa que indique que la conserva de higuerón con zanahoria tiene un tiempo superior de vida útil que la Norma INEN 419, por lo tanto, se puede considerar válido para el proceso de fabricación.

\section{CONCLUSIONES}

Mediante procesos tecnológicos de industrialización se pueden establecer que el método de procesamiento más viable para conservar las propiedades nutricionales abundantes del higuerón (Cucúrbita odorífera Vell) [1], con zanahoria amarilla (Daucus carota) es el desarrollo de elaboración de mermelada.

Las formas vegetativas de bacterias, levaduras y hongos se destruyen casi instantáneamente a temperaturas de cocción altas, que se comprueba mediante los análisis microbiológicos de la mermelada de higuerón con zanahoria amarilla, donde se comprobó que los productos mantuvieron una estabilidad microbiológica mínima de 4 meses. Se estableció un tiempo de vida útil aproximado de las conservas elaboradas alrededor de 6 meses, tiempo similar a lo planteado por Baixauli, el año 2015 [18] y Saavedra, el año 2015) [19] en procesamiento de mermeladas en diferentes frutas.

Los estándares de calidad del higuerón y zanahoria amarilla determinándose que la materia prima guardaba las condiciones óptimas necesarias para la elaboración de la mermelada en estudio, indicando que los tratamientos poseen estabilidad ya que fueron elaborados con la aplicación de los debidos parámetros de manufactura, respetando el tiempo y temperatura de procesamiento de elaboración.

Se aplicó un análisis sensorial para la aceptabilidad del producto elaborado que está acorde a los desarrollos de validación de vida útil por análisis sensorial [17] con muestras de 3 tratamientos de las 9 formulaciones para cada catador con su respectiva replica, determinando que el mejor tratamiento es el elaborado a partir de la formulación de higuerón 30\% y zanahoria $20 \%$.

\section{RESUMEN}

El objetivo de este estudio fue conocer el tiempo de vida útil en la elaboración de mermelada mediante una mezcla higuerón y zanahoria, motivado por el desconocimiento de un sinnúmero de especies de tubérculos y hortalizas, evidenciado por el bajo consumo de estos alimentos y la carencia de tecnología adecuada para el procesamiento del mismo y aprovechamiento de sus nutrientes. Las dos materias primas de alto contenido de vitaminas y minerales; el higuerón se considera como fruta-hortaliza por sus características y composición química, siendo posible la industrialización en productos semejantes a los elaborados con las frutas. La zanahoria es un alimento excelente desde el punto de vista nutricional por lo que aporta al mejoramiento de la calidad de la mermelada. Los tratamientos se mantuvieron bajo condiciones ambientales durante 40 días en los que se registró $\mathrm{pH}$ - sólidos solubles ( ${ }^{\circ}$ Brix) - acidez - análisis microbiológicos y pruebas organolépticas con la finalidad de identificar el mejor tratamiento experimental. De acuerdo a los resultados obtenidos y al análisis microbiológico se establece un tiempo de vida útil alrededor de 6 meses.

Palabras clave: Procesamiento de hortalizas; manufactura de hortalizas; higuerón; zanahoria; nutrientes; mermelada.

\section{BIBLIOGRAFÍA}

1. S. Zapata, A. M. Piedrahita y B. Rojano, "Oxygen radical scavenging capacity (ORAC) and total phenols fruit and 
vegetables from Colombia,» Perspectivas Nutr Humana, $2014 ; 16,(1): 25-36$.

2. R. Lira y I. Rodríguez, "Catalog of Mexico Cucurbitaceae family.,» Unidad de Biología. Tecnología y Prototipos, FES Iztacala, Base de datos SNIB - Conabio DS002, 2006. [En línea]. Available: www.conabio.gob.mx. [Último acceso: 12 Diciembre 2013].

3. M. Sanchéz-Hernández, C. Villanueva-Verduzco, C. Sanchéz-Hernandez, J. Sahugún-Castellanos y E. VillanuevaSánchez, "Response participatory selection of varieties of squash Sierra Norte de Puebla, Mexico,»Rev Chapingo Serie Horticultura, 2014; 20, (1) 41-56.

4. C. R. E. Garay y C. M. Z. Aguilar, "Evaluation of carrot varieties planted in summer,» Invest Agraria, 2013; 13, (2) 75-79.

5. Infoagro, «Vegetables / Carrots growing,» 2008. [En línea]. Available: http://www.infoagro.com/hortalizas/zanahoria. htm. [Último acceso: 13 Diciembre 2013].

6. FAO, Development of carrot and orange marmalade. InterAmerican Institute for Cooperation on Agriculture, San José: FAO, 2009.

7. E. Morales, "Using the yellow carrot (Daucus carota) to produce a fermented beverage,» Universidad Técnica de Ambato, Ambato, 2011.

8. L. Hidalgo, Horticulture notes, Riobamba: Escuela Politécnica del Chimborazo, 2008.

9. Bejo Zaden, «Carrots on the move,» Symposium Zanahorias Bejo Zaden, Warmenhuizen - Holanda, 2008.

10. Instituto Ecuatoriano de Normalización, Reporting Standards: INEN 419. (canned vegetables), Quito: INEN,
1979.

11. J. D. Vince Rodriguez, «Implementation of Systematic Continuous Improvement Methodology in a food factory,» Escuela Superior Politécnica del Litoral, Guayaquil, 2011.

12. R. Hernández, C. Fernández y P. Baptista, Investigation methodology, México: McGraw Hill, 2010.

13. R. Gordón-Mendoza y I. Camargo-Buitargo, "Selection for estimating statistical experimental precision in assays corn,» Agronomía Mesoamericana, 2015; 26, (1) 55-63.

14. Montgomerv y Douglas, Design and Analysis of Experiments, México: Editorial Limusa, 2004.

15. M. Santaella, C. M. Graciá, M. J. Periago y J. Santaella, "Sensory evaluation of different commercial presentations bream (Sparus aurata L.) aquaculture,» Anal Veterinaria Murcia; 2012, 28, 85-96.

16. M. N. Santosa, J. V. Orsine, I. D. A. Alexandre, R. Cañetec y M. Novaesd, "Sensory and physicochemical evaluation of Ipomoea batatas enriched with pro-vitamin in natura or processed,» J f Pharma. Pharmacognosy Res. 2014; 2, (4) $110-8$

17. V. Vásquez-Villalobos, J. Vásquez y E. Méndez, «New method for determining sensory life using fuzzy logic: If artichoke hearts (Cynara scolymus L.) marinated canned, 》 Scientia Agropecuaria, 2015; 6, (2) 99-109.

18. E. A. Baixauli, "Influence of different polyols in the physicochemical and sensory properties of strawberry jam,» Doctoral dissertation, Valencia, 2015.

19. L. Saavedra, "full use of passion fruit (Passiflora edulis flavicarpa) in extracting pectin and making jams,» Work degree, Quito, 2015. 